\title{
Le diagnostic du déficit androgénique lié à l'âge
}

\author{
Nelly MORTINIERA, Florence SCHLEICH, Jean-Jacques LEGROS
}

Service Universitaire d'Endocrinologie, CHU - Sart Tilman, Liège, Belgique

\section{RESUME}

Chez les hommes âgés, il existe une décroissance progressive du taux de testostérone.

Après $\mathbf{5 0}$ ans, la chute de ce taux est beaucoup plus marquée et peut entraîner une variété de signes cliniques incluant une déficience sexuelle, une baisse de la force musculaire, une anémie, des symptômes dépressifs.

Dans plusieurs cas, il reste à démontrer que ces symptômes sont la conséquence du déficit androgénique. Sous traitement substitutif, ces symptômes peuvent régresser. II existe une augmentation progressive de la SHBG (Sex Hormone Binding Globulin) avec l'âge. Le diagnostic de déficit androgénique nécessite de prendre en compte la forme bioactive de la testostérone (c'est-à-dire testostérone libre + testostérone liée à l'albumine). De nombreux tests ont été réalisés pour détecter la déficience androgénique. La plupart de ces tests ont une bonne sensibilité mais une faible spécificité ; ceci est dû à l'interférence de la dépression indépendamment des androgènes et du niveau de qualité de vie.

Mots clés : hommes âgés (plus de 50 ans), diminution de la testostérone, symptomatologie variéé, tests de détection faible spécificité

\section{INTRODUCTION}

Le terme d' «andropause " renvoie au vieillissement de l'axe hypothalamo-hypophyso-gonadique de l'homme par analogie avec la perte des menstruations chez la femme (" ménopause »). Ce vieillissement comporte une diminution des taux plasmatiques de testostérone biologiquement disponible, d'où la dénomination anglo-saxonne "Partial Androgen Deficiency of the Aging Male (PADAM) ou DALA (déficit androgénique lié à l'âge) plus correct chez l'homme. Le déficit androgénique peut induire une diversité de manifestations cliniques, dont la dépression, ainsi que la diminution de la force musculaire, de l'énergie, de la libido et de la fonction érectile [6]. L'étude des androgènes chez l'homme vieillissant tire également un intérêt du fait qu'un lien a été établi entre l'hypoandrogénisme et l'ostéoporose.

Plusieurs obstacles s'opposent à l'obtention d'un consensus quant à la définition de l'andropause. Les symptômes de l'andropause sont non seulement aspécifiques comme en témoigne l'énumération ci-dessus - mais en plus, à la différence des plaintes ménopausiques, ils s'installent progressivement et à un rythme variable d'un individu à l'autre : certains hommes présentent un déficit en testostérone dès l'âge de 40 ans, alors que d'autres entretiennent une fonction testiculaire normale à 80 ans ou plus. Enfin, dans nombre de cas, les symptômes pris en charge ne sont pas strictement attribuables au facteur gonadique, mais se révèlent d'origine multifactorielle (baisse des taux d'autres hormones - dont l'hormone de croissance, l'insulin like growth factor, la déhydroépiandrostérone -, vieillissement cellulaire général, etc.).

\footnotetext{
Correspondance :

Pr Jean-Jacques LEGROS - Service Universitaire d'Endocrinologie, CHU - Sart Tilman, B-4000 Liège. Belgique - Tel 003243667083 - Tel 003243667261 Email jean-jacques.legros@ulg.ac.be
} 
Chez l'homme adulte, la testostérone est synthétisée par les cellules de Leydig, logées dans le tissu interstitiel testiculaire. Cette synthèse est essentiellement placée sous le contrôle de l'hormone lutéinisante hypophysaire (LH), dont la sécrétion est réglée par la gonadolibérine hypothalamique (LHRH). A l'instar de la LH, la sécrétion de la testostérone dans le sang évolue selon un rythme circadien, avec un pic vers 8 à 9 heures du matin et des taux plus faibles en soirée.

Dans le sang, environ $40 \%$ de la testostérone circulante est puissamment liée à la sex hormone binding globulin (SHGB). Les quelque $54 \%$ liés à l'albumine et à d'autres protéines plasmatiques, ainsi qu'environ $2 \%$ de testostérone libre constituent la fraction biologiquement disponible. Le taux de testostérone totale se situe chez l'homme adulte entre 300 et $1000 \mathrm{ng} / \mathrm{dl}$.

Dans la périphérie, la testostérone est convertie en dihydrotestostérone (DHT) par la $5 \alpha$-réductase. Lors de la liaison de ces deux substances à leurs récepteurs respectifs, la testostérone régule la sécrétion des gonadotrophines, tandis que la DHT induit la plupart des effets androgènes à proprement parler.

La testostérone est essentielle au développement et au maintien des tissus reproducteurs et des caractères sexuels secondaires. Elle assure également le support de la masse corporelle maigre, de la taille des muscles et de la force. Par le biais de sa transformation en œstrogène, la testostérone stimule la formation osseuse. Au niveau psychologique, la testostérone semble être positivement liée à l'excitation sexuelle, à la confiance en soi et à la sociabilité.

\section{LE VIEILLISSEMENT DE L'AXE HYPOTHALAMO-HYPOPHYSO-TESTICULAIRE}

L'avancée en âge voit s'installer une diminution des taux sanguins de testostérone libre. Certains auteurs signalent l'amorce de ce phénomène à partir de l'âge de 40 ans [7] qui semble être attribuable à une raréfaction de cellules de Leydig. Une diminution de la perfusion testiculaire est observée.

En même temps s'installe une modification du fonctionnement hypothalamo-hypophysaire, comportant la perte des variations nycthémérales des taux de testostérone, une diminution de la fréquence des pics de LH de grande amplitude et une augmentation de la sensibilité des gonadotrophines au feedback négatif exercé par les hormones sexuelles [21]. Les taux de testostérone diminuent principalement dans la matinée et rejoignent, pour ainsi dire, les valeurs mesurées chez l'homme jeune pendant l'aprèsmidi.

Enfin, il y a lieu de signaler une augmentation de la production de sex hormone binding globulin, qui est un facteur essentiel de la diminution de la testostérone biologiquement disponible chez l'homme âgé $[6,16]$. L'on constate ainsi qu'avec l'âge, la testostérone libre chute plus rapidement que la testostérone totale [22]. L'augmentation des taux de la protéine de liaison s'explique, chez l'homme âgé, par la hausse du rapport estradiol/testostérone. En effet, si la production de testostérone par les cellules de Leydig diminue, l'estradiol dérive lui, en grande partie, de la transformation (aromatisation) périphérique des androgènes surrénaliens, dont la déhydroépiandrostérone.

L'obésité peut aggraver l'hypoandrogénisme [5], vraisemblablement en raison d'une aromatisation accrue, au sein du tissu adipeux, de la testostérone en estradiol, avec baisse secondaire de la $\mathrm{LH}$.

\section{MANIFESTATIONS LIEES AU DEFICIT EN TESTOSTERONE}

Un déficit en testostérone peut se traduire par la diminution ou la perte de la libido et de la fonction érectile, des caractères sexuels secondaires et de l'énergie, avec un effet négatif sur l'humeur $[15,17]$.

Du point de vue somatique, une augmentation de la masse graisseuse [18] est observée, ainsi qu'une diminution de la masse et de la force musculaires [20].

Les études montrent également que la suprématie relative des estrogènes exerce un effet néfaste sur le système vasculaire, et en particulier coronarien, de l'homme [8].

Enfin, la carence en androgènes réduit la densité osseuse de l'homme et favorise l'ostéoporose [1, 2]. Ce phénomène concerne davantage le rachis que le col du fémur. Dans ce contexte, il faut garder à l'esprit que les fractures de l'homme âgé provoquent une morbidité plus importante que celles de la femme de même âge [9]. Les études récentes suggèrent que l'effet favorable des androgènes sur le squelette de l'homme serait dû, en réalité, à l'aromatisation de ceux-ci en estrogènes. Or, s'il existe chez l'homme âgé une prédominance relative des estrogènes, il y a lieu de rappeler aussi que le taux biologiquement disponible de ces hormones diminue, principalement en raison de la hausse de la SHBG.

\section{CHEZ QUI SUSPECTER UN DEFICIT EN ANDROGENES ?}

Le déficit en androgènes peut provoquer chez l'homme un ensemble de manifestations cliniques qui sont énumérées dans le Tableau 1 [19]. Afin de faciliter le dépistage du syndrome de déficit en androgènes parmi les hommes présentant une ou plusieurs de ces manifestations, plusieurs questionnaires ont été développés. Parmi ceux-ci figure le questionnaire Androgen Deficiency in Aging Male (ADAM), publié en 2000 par Morley et al. [14]. Ce questionnaire à dix questions a été traduit par notre équipe et validé par la réalisation simultanée du dosage de la testostérone libre dans un groupe de 745 hommes de 50 à 70 ans. II existait entre le groupe dans lequel le test ADAM était positif et celui où il était négatif une différence significative du taux de testostérone libre $(p<0,005)$ (Tableau 2). En revanche, la testostérone totale, elle, n'était pas significativement différente. La version française du questionnaire ADAM est 
Tableau 2 : Valeurs basales de la testostérone libre calculée (FTC), de l'hormone folliculo-stimulante (FSH) et de l'hormone lutéinisante $(L H)$ selon la positivité $(A+)$ ou la négativité $(A-)$ du test $A D A M$. Les calculs statistiques sont tous corrigés pour l'effet de l'âge. On voit que seule la testostérone libre calculée (fTC) est significativement différente dans les deux groupes (selon Legros et Delhez, 2002)

\begin{tabular}{|c|c|c|c|c|c|c|}
\hline & & \multicolumn{5}{|c|}{ Score ADAM } \\
\hline & & \multicolumn{2}{|c|}{ A- $(n=177)$} & \multicolumn{2}{|c|}{$A+(n=568)$} & \multirow[t]{2}{*}{$\mathbf{P}$} \\
\hline & & Moyenne & SD & Moyenne & SD & \\
\hline LH & $\mathrm{mUl} / \mathrm{ml}$ & 6,40 & 4,43 & 6,31 & 4,08 & 0,311 \\
\hline FSH & $\mathrm{mUl} / \mathrm{ml}$ & 6,95 & 5,65 & 7,89 & 7,62 & 0,633 \\
\hline Testostérone totale & $\mu \mathrm{g} / \mathrm{l}$ & 4,62 & 1,56 & 4,21 & 2,38 & 0,065 \\
\hline $\begin{array}{l}\text { Testostérone libre } \\
\text { calculée ( }(T c)\end{array}$ & $\mathrm{ng} / \mathrm{l}$ & 71,5 & 26,62 & 63,50 & 22,15 & 0,005 \\
\hline
\end{tabular}

Tableau 1 : Manifestations cliniques pouvant être associées à un déficit en androgènes (selon Mathieu et al, [13]).

\section{Symptômes vasomoteurs et nerveux}

Bouffées de chaleur similaires à celles de la ménopause

Episodes de transpiration

Insomnie

Nervosité

Perturbations de l'humeur et des facultés cognitives

Irritabilité et léthargie

Diminution du bien-être

Manque de motivation

Energie mentale basse

Difficultés de mémoire à court terme

Symptômes dépressifs

Faible estime de soi

Anxiété inhabituelle

Virilité

Diminution de la vigueur et de l'énergie physique

Diminution de la masse musculaire et de la force

Perte de la pilosité

\section{Sexualité}

Diminution de l'intérêt ou du désir sexuel

Réduction de l'activité sexuelle

Faible fonction érectile

Qualité limitée de l'orgasme

Faible éjaculation

Diminution du volume de l'éjaculat

reproduite dans le Tableau 3 . Lorsque le résultat de ce test est positif, l'investigation ultérieure peut s'orienter vers la recherche et la mise en évidence d'un syndrome de déficit en androgènes.

Les manifestations somatiques de la carence androgénique pouvant éventuellement être décelées à l'examen physique comprennent la raréfaction de la pilosité pubienne, ainsi que le rétrécissement et la mollesse des testicules. La gynécomastie peut traduire la suprématie relative des estrogènes. Enfin, il est utile d'exclure l'existence d'une varicocèle, qui constitue une cause importante de perturbation de l'hormonogenèse [3].

Si le vieillissement de l'axe hypothalamo-hypophyso-testiculaire, éventuellement doublé de facteurs favorisants comme l'obésité et la varicocèle, offre certes une explication évidente au déficit en testostérone de l'homme de plus de 50 ans, il n'en reste pas moins important d'exclure également certaines autres causes possibles.

A cet égard, la distinction entre les formes primaires et secondaires d'hypogonadisme peut être utile, ceci par le biais du dosage de l'hormone folliculo-stimulante (FSH) et de l'hormone lutéinisante (LH). Chez l'homme vieillissant, chez qui l'hypogonadisme est essentiellement le fait d'une baisse primaire du fonctionnement des testicules, les taux de ces hormones sont en principe élevés (hypogonadisme hypergonadotrope). En revanche, ils sont faibles en cas d'hypogonadisme attribuable à une déficience hypothalamo-hypophysaire, également qualifié d'hypogonadotrope (Figures 1 et 2).

II faut signaler que l'on peut observer un hypogonadisme avec sécrétion gonadotrope relativement basse non seulement dans les atteintes hypothalamo-hypophysaires, mais aussi dans l'éthylisme chronique. En outre, il existe chez certains hommes âgés une hypersensibilité du gonostat, qui provoque des valeurs normales basses de $\mathrm{LH}$ face à une testostérone abaissée [4]. II apparaît également que certains patients à testostérone basse présentent des concentrations élevées en dérivés de pesticides, mais la relation reste à confirmer.

L'une des causes les plus fréquentes de déficience hypothalamo-hypophysaire pouvant être suspectées chez l'homme âgé est l'hyperprolactinémie. Celle-ci diminue les taux de FSH et de LH en freinant la libération hypothalamique de LHRH. Ses causes sont nombreuses (adénome hypophysaire, médicaments, dont les neuroleptiques, etc...). L'hyperprolactinémie provoque rarement une gynécomastie et une galactorrhée chez l'homme. 
Tableau 3 : Test ADAM (Androgen Deficiency of Aging Male). Version anglaise : Morley et al. [14] ; version française : Legros J.J., Delhez M. [11]. Le test est positif si le patient répond oui à la question 1 et/ou 7 ou à au moins 3 des autres questions.

\section{VERSION ANGLAISE}

1. Do you have a decrease in libido (sex drive) ?

2. Do you have a lack of energy?

3. Do you have a decrease in strength and/or endurance ?

4. Have you lost height?

5. Have you noticed a decreased 'enjoyment of life'?

6. Are you sad and/or grumpy?

7. Are your erections less strong ?

8. Have you noted a recent deterioration in your ability to play sport?

9. Are you falling asleep after dinner ?

10. Has there been a recent deterioration in your work performance?

\section{VERSION FRANCAISE}

1. Avez-vous constaté une diminution de votre libido (désir d'avoir des rapports sexuels) ?

2. Sentez-vous un manque d'énergie?

3. Avez-vous constaté une diminution de force musculaire et d'endurance à l'effort?

4. Avez-vous remarqué que vous aviez rapetissé ?

5. Avez-vous remarqué une diminution de votre joie de vivre?

6. Vous sentez-vous triste ou grincheux ?

7. Vos érections sont-elles moins fortes ?

8. Avez-vous remarqué une diminution de votre capacité de "faire du sport " ?

9. Tombez-vous endormi après les repas ?

10. Avez-vous remarqué une diminution récente de votre capacité de travail?

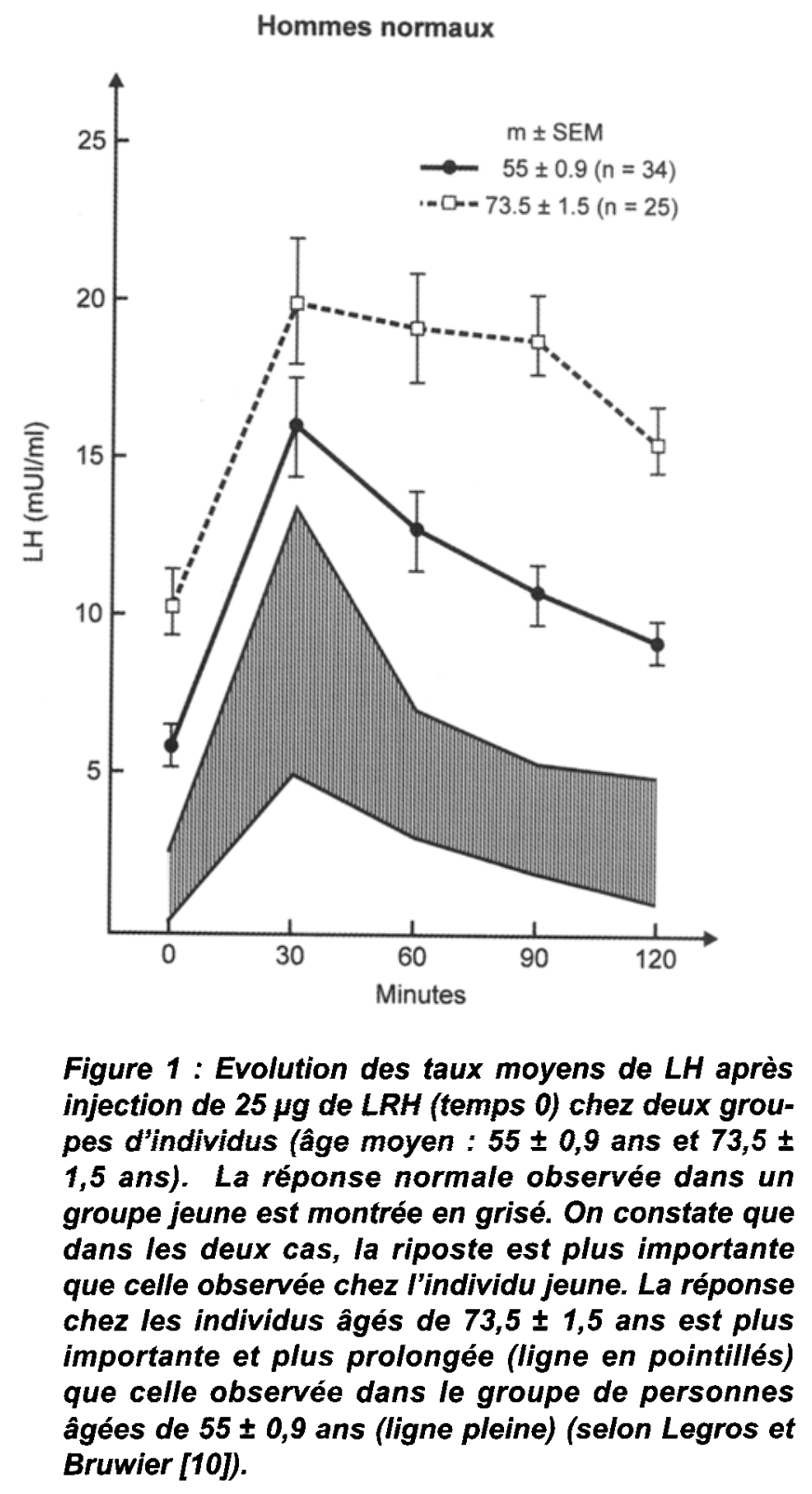

Figure 2 : Comparaison entre le taux de base de la testostérone plasmatique (mesurée par chromatographie en phase gazeuse après extraction) (en ordonnée) et les pics de $\mathbf{L H}$ (en abscisse) sous 25 $\mu g$ de LRH chez deux groupes de patients âgés, soit de 73,5 $\pm 1,5$ ans (carrés), soit de $55 \pm 0,9$ ans (points). On constate qu'il existe une relation entre la libération de la $\mathrm{LH}$ et le taux de testostérone dans le groupe le plus âgé. Cette relation est présente car les patients dont le taux de testostérone est le plus bas montrent des réponses en $L H$ très élevées. Ce type de relation n'est pas trouvé dans les groupes plus jeunes chez lesquels les taux de testostérone plasmatiques sont dans l'ensemble normaux (selon Legros et Bruwier [10]). 


\section{LE DOSAGE DE LA TESTOSTERONE}

Le dosage de la testostérone totale a longtemps été utilisé chez l'homme jeune. L'augmentation de la SHBG avec l'âge rend son utilisation problématique chez l'homme plus âgé. La première approche consiste en un dosage de testostérone, accompagné de l'estimation après dialyse de la testostérone libre. Cette technique de référence est longue et coûteuse et n'est que très exceptionnellement utilisée en routine clinique. Les dosages indirects de testostérone libre (méthode par résine) sont peu fiables et, en pratique, de meilleurs résultats sont obtenus par le calcul de la testostérone libre se basant sur la valeur de la testostérone totale et de la SHBG. Les valeurs ainsi obtenues sont étroitement corrélées avec les valeurs de testostérone " biodisponible » (libre et faiblement liée aux albumines) [23].

Les recommandations concernant le type de dosage de la testostérone à utiliser (totale, libre, biodisponible mesurée ou biodisponible calculée) sont envisagées au point $1 \mathrm{~b}$ du rapport du NIA et à la recommandation 4 de l'ISSAM. La testostérone étant sécrétée selon un rythme circadien, il est classiquement recommandé de réaliser les mesures à jeun entre 7 et $10 \mathrm{~h}$ du matin : notons toutefois que ces variations circadiennes s'atténuent progressivement avec le vieillissement. De plus, il est recommandé de répéter les mesures à 2 semaines d'intervalle étant donné les fluctuations spontanées des taux d'androgènes tant chez l'homme jeune que chez l'homme âgé [23]. Enfin, bien que la valeur de testostérone plasmatique ne soit pas influencée par la prise des repas, la présence des lipides dans le sang peut perturber une des étapes du dosage de la testostérone et il est donc recommandé que le patient soit à jeun pour la réalisation de la prise de sang.

Le dosage de la testostérone ne doit néanmoins pas faire oublier l'examen clinique classique. Dans une très belle étude récente, consacrée à une comparaison entre les bilans stéroïdiens périphériques et le volume testiculaire, Mahmoud et al. [12] ont démontré que le volume testiculaire (mesuré par ultrasonographie) est en relation avec la testostérone circulante : un volume moyen de $14,3 \mathrm{ml}$ permet de prédire une valeur anormalement basse de testostérone biodisponible avec une spécificité tout à fait satisfaisante de $79 \%$ mais une sensibilité relativement faible de $46 \%$.

\section{LES TESTS PSYCHOLOGIQUES}

Plusieurs tests ont été proposés afin de permettre d'établir un diagnostic vraisemblable d'uandropause " sans avoir à réaliser les dosages de testostérone, en particulier de testostérone biodisponible. D'une façon générale, on pourra retenir que ces tests, souvent longs et difficiles à remplir, sont de peu d'intérêt pour le diagnostic de la déficience androgénique mais ils peuvent par contre être d'un grand intérêt pour le suivi d'un traitement androgénique substitutif.

Un questionnaire simplifié de détection de l'andropause, avait également été développé par Morley et collaborateurs [14] (St Louis Androgen Deficiency in Aging Male, ADAM) dont nous avons validé la version française en Pro- vince de Liège. Le test est considéré comme positif si le patient répond oui à la question 1 et/ou 7 ou au moins à 3 des autres questions. De septembre 2000 à avril 2001, 745 hommes (âge moyen : $59,6 \pm 5,9$ ans) se sont présentés dans le cadre d'une campagne de dépistage. Ils ont subi, à jeun, avant $10 \mathrm{~h}$ du matin, une prise de sang pour dosage de testostérone totale, de SHBG (permettant le calcul de la testostérone libre, $\mathrm{fTc}$ ) de FSH et de $\mathrm{LH}$, le test ADAM était rempli peu après la prise de sang. Le test était positif chez 568 et négatif chez 177 patients. L'âge moyen des hommes présentant un test positif $(60,1 \pm 6,1$ ans) est légèrement mais significativement plus élevé que celui des patients ayant un test négatif $(57,6 \pm 5,7$ ans $)(p<0,001)$ : il est donc important dans ce type d'étude de corriger les valeurs pour l'âge.

Malgré cette correction, on constate que la testostérone libre calculée est significativement plus basse chez les patients dont le test Adam est positif que chez les patients dont le test Adam est négatif (Figure 3 ) alors que la testos-
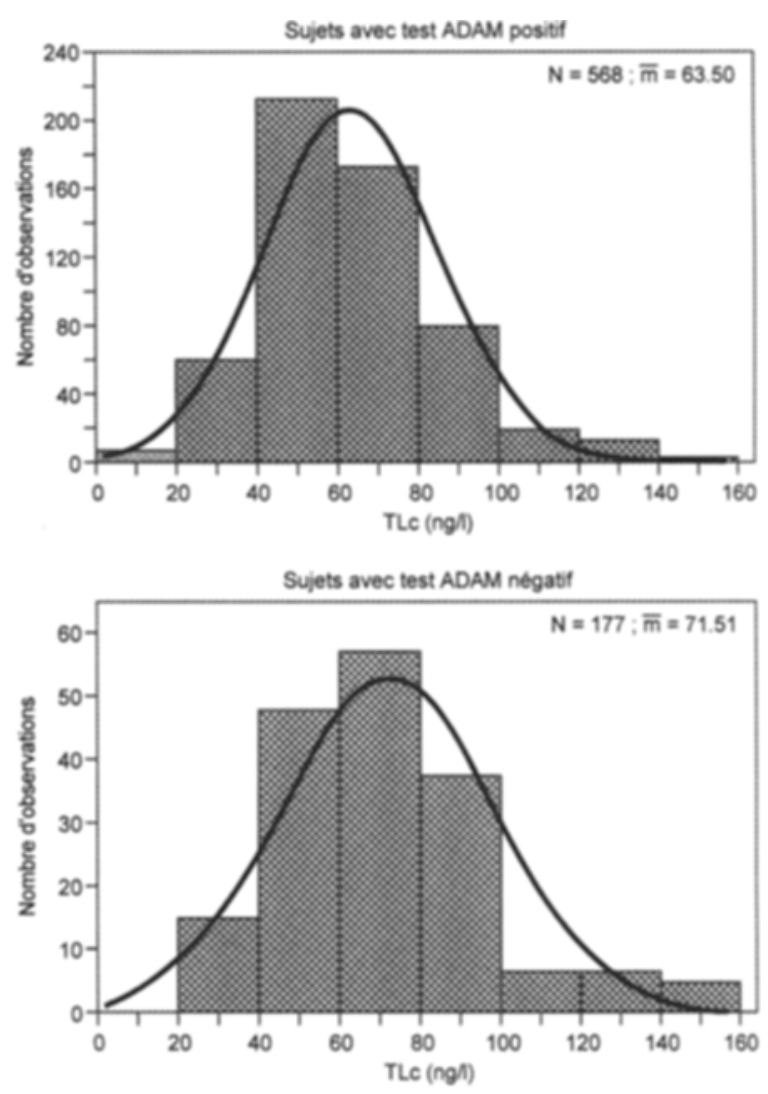

Figure 3 : Histogramme des valeurs de testostérone libre calculée. Ces valeurs (en rectangle) sont comparées à la répartition théorique (ligne noire) chez 745 hommes âgés de 59,6 $\pm 5,9$ ans selon que le test ADAM positif $(n=568)$ ou négatif (normal) (en bas) présente une répartition des dosages de la testostérone libre calculée (fTc) comparable à la répartition théorique des valeurs, ce qui n'est pas le cas dans le groupe dont le test ADAM est positif (anormal) (en haut). Bien que la valeur moyenne de fTC soit significativement plus basse dans le groupe A+. On note un important chevauchement des valeurs, ce qui limite l'application clinique pratique de cette démonstration (selon Legros et Delhez [10]). 
térone totale, la $\mathrm{LH}$ et la FSH ne sont pas significativement différentes. La recherche d'une valeur seuil amenant à une sensibilité optimale de l'ADAM a permis de retenir la valeur de $70 \mathrm{ng} / \mathrm{l}$ de testostérone libre calculée donnant une sensibilité de $80,1 \%$ mais une spécificité faible de $32,2 \%$.

Le choix de valeurs de testostérone libre calculée plus élevées permet d'augmenter légèrement la spécificité à $34,3 \%$ mais au prix d'une moindre sensibilité à $77,9 \%$.

Ces résultats sont donc différents de ceux obtenus par Morley et ses collaborateurs puisque ces auteurs décrivent une sensibilité du même ordre que la nôtre à $88 \%$ mais une spécificité nettement meilleure de $60 \%$. II est vraisemblable qu'une partie de cette discordance est due au fait que les populations testées étaient différentes : médecins canadiens en activité dans l'étude initiale de Morley, hommes pour la plupart retraités dans notre étude, d'autre part. L'interférence des phénomènes dépressifs indépendant des fluctuations androgéniques est importante. En effet, dans une étude plus spécifique et reprenant 153 sujets triés, et d'où étaient exclues les personnes utilisant chroniquement des anti-dépresseurs ou des anxiolytiques, nous avons pu démontrer que le test ADAM était faussement positif (c'est-à-dire positif face à une testostérone libre de plus de $70 \mathrm{ng} / \mathrm{l}$ ) chez les patients fortement dépressifs indépendamment de toute pathologie de l'imprégnation androgénique. Nous considérons donc que ce questionnaire n'est pas spécifique à la déficience androgénique mais qu'il constitue un élément de dépistage psychoendocrinien précieux facile à remplir et pouvant permettre une approche clinique et biologique du patient de plus de 50 ans selon un arbre décisionnel montré au Tableau 4 [11].

A titre informatif, les résultats obtenus combinant le dosage de la testostérone libre calculée, la FSH, la LH ainsi que la cotation du test ADAM lors d'une campagne de dépistage dans la Province de Liège portant sur près de 6000 hommes âgés de 50 à 70 ans sont montrés à la Figure 1 . On y constate que plus de $50 \%$ des hommes se présentant pour ce dépistage présentent des bilans biologiques et un test ADAM normaux. De 10 à $15 \%$ des patients présentent un bilan biologique strictement normal avec un test ADAM fortement positif : ils sont très suspects de troubles dépressifs. On notera également 10 à $15 \%$ de patients présentant un hypogonadisme hypergonadotrope (portant sur la FSH et parfois sur la $\mathrm{LH}$ ), ce qui correspond au pourcentage de patients présentant un tel hypogonadisme dans une consultation interdisciplinaire spécialisée (The Aging Male Clinic, 2002).

\section{CONCLUSION}

L'avancée en âge comporte chez l'homme une diminution perceptible des taux de testostérone libre. Après l'âge de $\mathbf{5 0}$ ans, le déficit androgénique peut devenir très important, en induisant ainsi une symptomatologie clinique. Des questionnaires simples sont disponibles pour dépister ces patients et donner lieu à la documentation du déficit androgénique par le dosage de la testostérone libre. Insistons sur le fait que ces
Tableau 4 : Arbre décisionnel au départ de la réalisation du test ADAM (Legros et Delhez [11]).

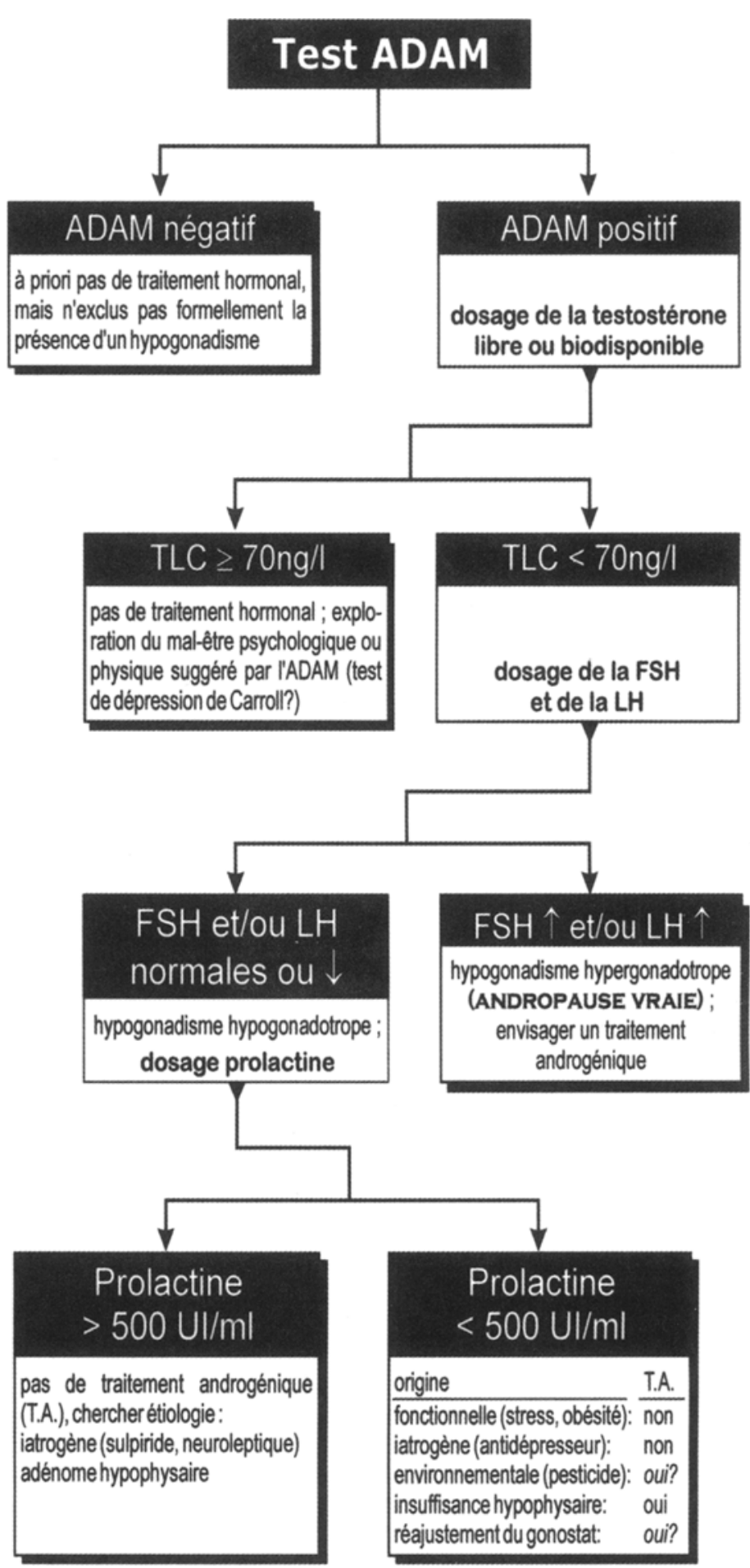

questionnaires sont peu spécifiques et fortement influencés par les problèmes dépressifs ou de " moindre qualité de vie ". Le diagnostic d'une déficience androgénique liée à l'âge (DALA) une fois établi par l'adjonction d'un bilan de fonctionnement hypothalamo-hypophysaire, un traitement substitutif peut être envisagé, après prise en compte des contre-indica- 
tions (voir article Kuhn et Thorin-Savoure, présent numéro d'Andrologie). L'évaluation du résultat clinique tiendra compte du fait que la symptomatologie associée à l'andropause est souvent attribuable à un mécanisme multifactoriel débordant le seul cadre du vieillissement hypothalamo-hypophyso-gonadique.

\section{REFERENCES}

1. BOONEN S., VANDERSCHUEREN D., BOUILLON R. : Ouderdomsgebonden androgeendeficiëntie en heupfacturen bij de bejaarde man. Tijdschr. Geneesk., 1997, $53: 1584$.

2. BOONEN S., VANDERSCHUEREN D., GEUSENS P., BOUILLON R. : Age-associated endocrine deficiencies as potential determinants of femoral neck (type II) osteroporotic fracture occurrence in elderly men. Int. J. Androl., 1997, 20:134.

3. COMHAIRE F., VERMEULEN, A. : Plasma testosterone in patients with varicocele and sexual inadequacy. J. Clin. Endocrinol. Metab., 1975, $40: 824$.

4. DESLYPERE J.P., KAUFMAN J.M., VERMEULEN T. et al. : Influence of age on pulsatile luteinizing hormone release and responsiveness of the gonadotrophs to sex hormone feedback in men. J. Clin. Endocr. Metab., 1987, 64 : 68-73.

5. GIAGULLI V.A., KAUFMAN J.M., VERMEULEN A. : Pathogenesis of decreased androgen levels in obese man. J. Clin. Endocrinol. Metab., 1994, 79 : 997.

6. GOOREN L. : Endocrine aspects of ageing in the male. Mol. Cell Endocrinol., 1998,145: 153-159.

7. GRIFFIN J.E., WLSON J.D. : Pathologie des testicules. In : McGraw-Hill. ed. Medecine Interne. Harrison, 14e edition, 2000.

8. GUTAI J., LA PORTE R., KULLER L. et al. : Plasma testosterone, high density lipoprotein cholesterol and other lipoprotein fractions. Am. J. Cardiol., 1981, $48: 897$.

9. KAMEL H.K., PERRY H.M., MORLEY J.E. : Hormone replacement therapy and fracture in older adults. J. Am. Geriatr. Soc. 2001, $49: 2179-2187$.

10. LEGROS J.J., BRUWIER M. : Vieillissement des systèmes de contrôle: les glandes endocrines. In : Bourlière $M$. ed. Précis de Gérontologie. Paris, Flammarion, 1982 : 62-80.

11. LEGROS J.J., DELHEZ M. : Détection de la déficience androgénique chez l'homme de plus de 50 ans : utilisation d'une version française du test ADAM. Med. Hyg., 2002, 60 : 14901495.

12. MAHMOUD A.M., GOEMAERE S., EL-GAREM Y., Van POTTELBERGH I., COMHAIRE F.H., KAUFMAN J.M. : Testicular volume in relation to hormonal indices of gonadal function in Community-Dwelling Elderly Men. J. Clin. Endocrinol. Metab. $2003,88: 179-184$

13. MATHIEU A., BRUWIER M., ALLOUCH A., ANDRIANNE R., LEGROS J.J. : Indications et contre-indications du traitement par androgènes chez l'homme de plus de 50 ans. Med. Hyg., 2001, $59: 1610-1614$.

14. MORLEY J.E., CHARLTON E., PATRICK P. et al. : 3rd. Validation of a screening questionnaire for androgen deficiency in aging males. Metabolism, 2000, $49:$ 1239-1242.
15. MORLEY J.E., PERRY H.M. : Androgen replacement therapy. J. Lab. Clin. Med., 2000, $135: 370-378$.

16. MORLEY J.E. : Endocrine aspects of ageing in the male. Maturitas, 2001, $38: 61-71$.

17. STERNBACH H. : Age-associated testosterone decline in men: clinical issues for psychiatry. Am. J. Psychiatry, 1998, 155: $1310-1318$.

18. TENOVER J.L. : Testosterone and the aging male. J. Androl., 1997, $18: 103-106$.

19. TREMBLAY R.R., MORALES A. : Canadian practice recommandations for screening, monitoring and treating men affected by andropause or partial androgen deficiency. The Aging Male, 1998, $1: 213-218$.

20. URBAN R.J., BODENBURG Y.H., GILKISON C et al. : Testosterone administration to elderly men increases skeletal muscle strength and protein synthesis. Am. J. Physiol., 1995, 269: E820-826.

21. VERMEULEN A., KAUFMAN J.M. : Aging of the hypothalamopituitary-testicular axis in men. Horm., 1995, 43 : 25-28.

22. VERMEULEN A., VERDONCK L., KAUFMAN J.M. : A critical evaluation of simple methods for the estimation of free testosterone in serum. J. Clin. Endocrinol. Metab., 1999, 84 : 36663672.

23. VERMEULEN A., KAUFMAN J.M. : Diagnosis of hypogonadism in the aging male. The Aging Male, 2002, 5 : 170-176.

Communication au $\mathrm{XX}^{\circ}$ Congrès de la Société d'Andrologie de Langue Française, Orléans, 11 - 13 décembre 2003.

Manuscrit reçu : janvier 2004 ; accepté février 2004.

\section{ABSTRACT}

The diagnosis of age-related androgen deficiency

Nelly MORTINIERA, Florence SCHLEICH, Jean-Jacques LEGROS

A progressive decrease of mean testosterone levels is observed in aging males. After the age of 50, this decrease is more marked and can lead to a variety of clinical symptoms including sexual deficiency, but also loss of muscle strength, anaemia, and depression.

However, in many cases it has not been formally demonstrated that these symptoms are causally related to androgen deficiency, but androgen replacement therapy is able to reverse these symptoms. 
Since there is a progressive increase of SHBG (Sex Hormone Binding Globulin) with age, demonstration of androgen deficiency must take into account only the bioactive form (i.e. free testosterone + albumin-bound testosterone).

Many screening tests designed to detect androgen deficiency have been proposed (ADAM, AMS, ...) : most of these tests are sensitive but poorly specific due to interference of androgen-independent depression and quality of life.

Key words : androgen deficiency, aging male, variety of clinical symptoms, many screening tests 